\title{
LA IMPLANTACIÓN DE EQUIPOS AUTODIRIGIDOS (EAD). UN ESTUDIO LONGITUDINAL EN UNA EMPRESA MANUFACTURERA DEL VALLE DE MÉXICO
}

\author{
José Vili Martínez González*, Damaris Mejía López**
}

\begin{abstract}
Martínez-González J.V., Mejía-López D. La implantación de equipos autodirigidos (EAD). Un estudio longitudinal en una empresa manufacturera del Valle de México. Hitos de Ciencias Económico Administrativas 2013;19 (53):33-38.
\end{abstract}

\section{RESUMEN}

Con gran frecuencia es posible observar la aplicación de programas enfocado al desarrollo de equipos autodirigidos en las organizaciones; sin embargo, en ocasiones, estos esfuerzos terminan siendo «un programa más» y se abona a la gran cantidad de esfuerzos realizados en la búsqueda de la tan ansiada productividad. Si bien, el fracaso de estos programas resulta multifactorial, existen casos de éxito que pueden coadyuvar a sentar las bases para encontrar una metodología que pueda extrapolarse a otras empresas colaborando con ello a su desarrollo y a incrementar el acervo de conocimientos en el campo de la administración. La presente investigación no experimental, longitudinal, descriptiva presenta la implantación exitosa de un programa de equipos autodirigidos en una organización manufacturera de 300 empleados ubicada en el Valle de México.

\section{Martínez-González J.V., Mejía-López D. The implementation of self-directed teams. A longitudinal study in a manufacturing company in the Valley of Mexico. Hitos de Ciencias Económico Administrativas 2013;19 (53):33-38. \\ ABSTRACT}

Very often it is possible to observe the establishment of programs aimed at developing self-directed teams in organizations, however, sometimes these efforts end up being "one more program» and so they are added to the many efforts made in the search for such longed productivity. While the failure of these programs is multifactorial, there are success stories that can help to lay the foundation of a methodology that can be extrapolated to other companies in order to collaborate to their development and increase the stock of knowledge in the field of administration. This non experimental, longitudinal, and descriptive research shows the successful implementation of a self-directed teams program in a manufacturing organization of 300 employees located in the Valley of Mexico.

Palabras clave: No experimental. Longitudinal.

Key words: Non experimental.Longitudinal. Descriptive. Descriptivo.

DIRECCIÓN PARA RECIBIR CORRESPONDENCIA: Correo electrónico: excepcional777@hotmail.com

xisten condiciones básicas para que el hombre sea la especie dominante en este mundo, algunas de ellas son su capacidad para aprender, para modificar su entorno y para trabajar en equipo. Esta última característica es la materia que compete a esta investigación; si bien el trabajar en equipo ha permitido el desarrollarnos y crecer como especie en estos momentos las exigencias del mundo empresarial, académico, deportivo y cualquier otra organización humana requiere que dichos grupos obtengan un desempeño de orden superior donde la jerarquía de abajo hacia arriba ya no es suficiente.

En este sentido, se conceptualizará lo que es un grupo. Para Robbins (1998) un grupo consiste en dos o más individuos que interactúan y son interdependientes los cuales se han reunido para el logro de objetivos particulares.

\footnotetext{
* Doctor en Administración. Profesor-Investigador. Universidad Nacional Autónoma de México.

** Profesora-Investigadora. Universidad Nacional Autónoma de México.
} 
Ahora bien, para Furnham (2002) existen cuatro características que permiten identificar a los grupos:

1. Están conformados por dos o más personas que tienen interacción social y son capaces de influir mutuamente en sus creencias y comportamientos;

2. Poseen objetivos, metas y propósitos comunes y aceptados;

3. Cuentan con una estructura relativamente estable, reglas y funciones que perduran a lo largo del tiempo y en diferentes situaciones sociales;

\section{Se perciben y reconocen abiertamente como grupo.}

En este orden de ideas McShane y Glinow (2005) mencionan que los equipos son un conjunto de dos o más personas que interactúan y ejercen influencia entre sí, se consideran igualmente responsables del logro de las metas comunes asociadas a los objetivos organizacionales y se perciben a sí mismos como una entidad social dentro de la organización.

Para esta investigación, se conceptualizará a los equipos autodirigidos (EAD) como:

Un grupo pequeño de personas (considerando de 2 a 15 integrantes) quienes poseen conocimientos y habilidades complementarios quienes conforman una unidad básica de trabajo capaz de resolver los requerimientos propios de su posición, de tal forma que cuando un observador externo mira su desempeño no le es posible identificar rangos ni autoridad predominantes, pues todos se encuentran trabajando en forma autónoma y comprometida con miras a un objetivo que todos conocen y comprenden que esta responsabilidad va más allá de solo entregar un producto o servicio, por lo que se hacen responsables del trabajo hasta su disposición final presentando siempre una comunicación abierta y franca.

Bauer (2002) menciona que los EAD han permitido que las empresas reporten beneficios como mejoras en la calidad de sus productos o servicios, costos de operación bajos, menor cantidad de administradores en los niveles intermedios, reducción de costos al tener una plantilla de personal reducida y solución de problemas con mayor rapidez. Al mismo tiempo han reportado que sus integrantes manifiestan mayor satisfacción en sus empleos y mejoras en los índices de estrés al no estar sometidos a supervisión directa en su trabajo, desarrollando su creatividad y un sentido de eficiencia personal. En consecuencia las personas generan un mayor compromiso con la organización.

Davis y Newstrom (2002) parecen apoyar lo anterior al mencionar que otras ventajas que presentan las organizaciones que emplean una estructura con base en EAD son:

1. Mayor flexibilidad del personal;

2. Obtención de operaciones más eficaces gracias a la reducción de número de clasificaciones de puestos;

3. Disminución en las tasas de ausentismo y rotación de personal;

4. Mayores niveles de compromiso organizacional y satisfacción en el trabajo.

Con estos antecedentes y la búsqueda de lograr empresas cada vez más «planas» con los consecuentes ahorros y utilidades, pareciera que los EAD son la respuesta que toda empresa desea; sin embargo, las organizaciones que los han integrado mencionan que pueden requerirse varios años para que los equipos logren estabilizarse y obtener su potencial máximo, esto a su vez requiere de inversiones importantes y un compromiso claro con su implantación. En este mismo orden de ideas, existen algunos valores o tradiciones que ponen énfasis en el individualismo y obstaculizan su implantación, si a ello se le suma que existen descripciones de puesto rígidas y protegidas, el que los gerentes suelen sentirse amenazados por la pérdida de control y de seguridad personal en su trabajo se podrá ver que el camino para implantar los EAD no resulta fácil.

\section{Implantación de EAD en una empresa manufacturera del Valle de México}

La unidad de estudio a la que hace referencia esta investigación es una empresa manufacturera de tamaño mediano.

Antecedentes. La unidad de estudio presentó un cambio en su dirección general, el director decidió que para lograr los objetivos de crecimiento y consolidación que le ha encomendado, la junta de accionistas debe realizar varios cambios siendo uno de estos la implantación de EAD en la organización. Para ello, desarrolla en conjunto con su equipo de trabajo un programa donde se establece una serie de puntos necesarios para lograrlo, estos fueron: 
1. Lograr el compromiso de la alta dirección. Esto incluía a las 5 direcciones (Recursos Humanos, Finanzas, Producción, Logística, Mercadotecnia). En este punto, se resaltó la importancia de lograr el compromiso personal de cada uno de los directores con el proyecto de EAD.

2. Asignación de un presupuesto. Este punto resulta destacable, pues no solo se desarrollo el plan de actividades y la ruta crítica para el mismo sino que se asignaron los recursos económicos necesarios para su desarrollo.

3. Mapeo de procesos para poder identificar fronteras para el desempeño de los EAD, lo que permitiría desarrollar «autonomía con fronteras identificables» con la finalidad de lograr el control y al mismo tiempo excluir equipos que no podrían trabajar bajo este esquema.

4. Desarrollo de las nuevas descripciones de puesto para la realización de los EAD.

5. Obtener un diagnóstico de la condición actual de la organización en dos áreas (clima laboral y liderazgo).

En esta primera etapa que parecía quizá la más controlable, presentó una serie de complicaciones dentro de las que se destacaron:

1.- Lograr el compromiso de la alta dirección resulto mucho más complejo de lo que se esperaba, ya que la resistencia al cambio y los augurios de mala suerte fueron el común denominador de la alta dirección, para ello fue necesario el realizar acciones como juntas de sensibilización y alineación de objetivos, un curso de capacitación dirigido específicamente a la alta dirección para comprender la importancia de los EAD y como se implantarían y finalmente el tomar un liderazgo fuerte para llevar adelante el proyecto. Es importante, mencionar que uno de los elementos que presentó mayor resistencia por parte de los directivos fue la pérdida de estatus en la implantación de los EAD, en este sentido, Martínez (2001) menciona que los símbolos de poder llegan a ser más que parte de la vida de las personas, pueden llegar a ser la vida misma, por lo que para algunos directivos el pensar en perder el control de los procesos y mandar a las personas les resultaba casi inaceptable.
2.- El lograr la aprobación del presupuesto también resultó complejo, ya que la junta de accionistas veían el proyecto como un gasto en tiempos de crisis y resulto difícil la gestión de los recursos que además se vieron recortados del programa original.

3.- El mapeo de procesos reveló que se tenían muchas áreas de procesos sin control y aun más, existían muchas operaciones no necesarias que se realizaban simplemente porque así se habían estado llevando «desde siempre».

4.- El desarrollar las nuevas descripciones de puesto no solo fue complejo considerando el punto anterior, sino que además se anexaron las objeciones del sindicato que veía el proyecto como una amenaza a las posiciones que tenía dentro de la organización. Fueron necesarias muchas horas de negociación para explicar que de no realizar el proyecto la fuente de empleo desaparecería, todo ello para contar con su «aceptación condicionada» y con la observación de que estarían atentos a cada paso del proyecto.

5.- El encontrar instrumentos confiables y válidos que ayudarán a obtener el diagnóstico del clima laboral y liderazgo, también resultó complejo debido a que en el mercado se ofertaba una gran cantidad de posibilidades lo cual fué difícil encontrar una que brindara la certeza de ser útil y hubiera sido desarrollada para mexicanos. Finalmente, se consiguieron instrumentos que poseían validez y confiabilidad adecuada contando el primero con una Alpha Cronbach de 0.9774 y el segundo de 0.9364 .

Una vez solventada la primera etapa la cual fue mucho más tardada de lo esperado (el director pensó que podía realizarla en un periodo de 15 días y se extendió a los dos primeros meses de su gestión), se dio paso a la etapa dos que presentó los siguientes pasos:

1. Obtención del clima laboral y los estilos de liderazgo aplicando dichos instrumentos al total de los integrantes de la organización.

2. Realización de los cursos enfocados al proceso de implantación de los EAD.

3. Desarrollo de los indicadores para controlar el proceso de aplicación de los EAD.

4. Realización de los programas enfocados a la aplicación de los EAD en la organización marcando tiempos y responsables. 
Esta etapa también presentó complicaciones tales como:

1. La aplicación de los instrumentos se vio obstaculizada por:

a) Falta de coordinación para que el personal se presentara;

b) Resistencia por parte de algunos sectores de la empresa para participar en la actividad.

c) Falta por momentos de espacios para la aplicación del instrumento.

2.- Los cursos resultaron ser un impulso que permitió mejorar la comunicación, compartir una visión global, así como objetivos y metas, generando la comprensión de que la responsabilidad de la «cuna a la tumba» de los procesos, es de todos los integrantes de la organización, coadyuvando a mejorar la percepción de los participantes para enfocar los esfuerzos encaminados a la implantación de los EAD.

3.- El desarrollo de los indicadores resultó más complicado de lo que se esperaba, pues se llegó a considerar que se utilizarían para marcar nuevos estándares de producción y no solo como parte del proceso de aplicación de los EAD, por ello se requirieron de múltiples reuniones y logro de acuerdos.

4.- La programación que en un principio se pensaba sería en forma genérica se tuvo que segmentar por áreas para lograr su implantación ya que resultaba complejo el hacerlo al mismo tiempo en todas áreas, para ello, se dividió a la planta en tres criterios, áreas de aplicación inmediata (para seleccionarla se utilizaron los resultados tanto del mapeo como de los instrumentos), una vez que se tuviera avances significativos se continuaría con las áreas consideradas de aplicación media y al lograr avances significativos se avanzaría en las últimas áreas. Se consideraron tripulaciones de 2 a 15 integrantes para cada EAD.

Cuadro de control de la primera etapa (se presenta un resumen)

En el tema se comparten algunos de los principales indicadores que se seleccionaron para controlar el proceso de implantación de EAD (Los datos corresponden al periodo anual inmediato anterior al inicio del proyecto).
Se presentan 5 columnas (correspondiente a las direcciones) en las cuales se estructuró toda la organización. A la izquierda de cada columna se presenta los índices de la unidad de estudio al principio del proyecto y a la derecha los índices deseados al término del mismo.

Recursos humanos $(\mathrm{RH})$, incluye todas las actividades relacionadas con la administración del personal, funciones de higiene, seguridad, ecología, servicios generales y seguridad patrimonial.

Finanzas $(F)$, incluye todo lo relacionado con los temas de dinero desde pago a proveedores, clientes, autoridades, etc.

Producción $(\mathrm{P})$, considera la realización del producto desde la recepción de la materia prima por parte del almacén hasta el retorno del producto terminado al mismo, así como lo relacionado a mantenimiento.

Logística (L), responsable de la materia prima desde su selección hasta que producción la toma y nuevamente desde que producción la regresa como producto terminado hasta su distribución a los centros de distribución (incluyendo el transporte).

Mercadotecnia (M), tiene a su cargo todos los aspectos relacionados con la venta y crecimiento de la marca y lo relacionado a la calidad.

Dentro de las áreas de oportunidad se identifican las obtenidas por los instrumentos de medición de Clima Laboral (AOC) y Liderazgo (AOL), el primer instrumento aplicado a toda la organización y el segundo a los directores, gerentes, jefes y coordinadores, en la columna derecha lo que se desea lograr al término del proyecto estos se clasifican en Bajo (B), Medio (M) o Alto (A) (ver tabla I).

Como puede observarse, la aplicación de los EAD no resulta una tarea sencilla pero el éxito logrado por la empresa ha rendido entre otros los siguientes frutos:

a) Mejoras significativas en productividad, calidad y obtención de ahorros al disminuir en forma palpable mermas y mejorar los procesos.

b) Una mejora real en el clima laboral de la empresa en todas las áreas.

c) Mejoras en la creatividad y resolución de problemas. 
TABLA I. CUADRO DE CONTROL

\begin{tabular}{|c|c|c|c|c|c|c|c|c|c|c|}
\hline \multirow{2}{*}{$\frac{\text { Tema }}{\text { Rotación del personal en \% }}$} & \multicolumn{2}{|c|}{$\mathrm{RH}$} & \multicolumn{2}{|c|}{$\mathrm{F}$} & \multicolumn{2}{|c|}{$\mathrm{P}$} & \multicolumn{2}{|c|}{ L } & \multicolumn{2}{|c|}{$M$} \\
\hline & 5 & 2 & 2 & 1 & 60 & 20 & 62 & 20 & 38 & 10 \\
\hline $\begin{array}{l}\text { Mermas identificadas de sus } \\
\text { recursos asignados en \% }\end{array}$ & 12 & 2 & 6 & 1 & 13 & 5 & 12 & 5 & 14 & 5 \\
\hline Número de accidentes & 3 & 0 & 0 & 0 & 22 & 0 & 18 & 0 & 4 & 0 \\
\hline Reclamación de calidad & 0 & 0 & 0 & 0 & 36 & 0 & 42 & 0 & 64 & 0 \\
\hline AOL comunicación & $\mathbf{M}$ & A & M & A & B & A & $\mathbf{M}$ & $A$ & B & A \\
\hline $\begin{array}{l}\text { AOL capacidad para } \\
\text { relacionarse efectivamente }\end{array}$ & $\mathbf{M}$ & A & $\mathbf{M}$ & A & B & $A$ & $\mathbf{M}$ & $A$ & $\mathbf{M}$ & A \\
\hline AOL autocontrol & A & $A$ & A & $A$ & B & $A$ & $\mathbf{M}$ & $A$ & B & A \\
\hline $\begin{array}{l}\text { AOL abierto a nuevas } \\
\text { experiencias }\end{array}$ & $\mathbf{M}$ & A & B & $A$ & B & $A$ & $\mathbf{M}$ & $A$ & A & A \\
\hline AOCliderazgo & $M$ & A & $M$ & $A$ & B & A & $\mathbf{M}$ & A & B & A \\
\hline AOC Comunicación & M & A & M & A & M & A & $\mathbf{M}$ & A & B & A \\
\hline $\begin{array}{l}\text { AOC satisfacción hacia el } \\
\text { cambio }\end{array}$ & M & A & $\mathbf{M}$ & A & B & $A$ & $\mathbf{M}$ & $A$ & B & A \\
\hline AOC trabajo en equipo & M & A & $\mathbf{M}$ & $A$ & B & $A$ & $\mathbf{M}$ & $A$ & B & A \\
\hline
\end{tabular}

d) Mejoras reales en la atención de los clientes.

e) Desarrollo de procesos más eficientes.

f) Obtención de las certificaciones que se habían planteado como meta.

g) Una mejora en las relaciones con el sindicato.

h) Una disminución importante en la rotación del personal.

i) Una disminución casi total del índice de absentismo.

Si bien, quedan muchos retos y tiempo para madurar sus procesos para implementar los EAD, en esta empresa el rumbo se ve altamente prometedor. Han aprendido mucho en estos tres años y también han tenido que resolver problemas como:

a) La renuncia de uno de los directivos que no logró adaptarse a la forma en que se desarrollan los EAD.

b)Fue necesario resolver conflictos en algunos procesos donde no se habían delimitado claramente las fronteras.
Algunas de las ventajas que fueron de gran aplicación para el desarrollo de los EAD fueron:

a) Los resultados de los instrumentos que permitieron encontrar las áreas de oportunidad.

b)La realización de los cursos (que ahora son constantes) que permitieron mejorar los procesos de comunicación.

c) La utilización del mapeo de procesos que permitieron delinear fronteras.

d) El desarrollo de indicadores que permitió el conocer como se realizó el proceso.

Para tener un marco comparativo con respecto al inicio del proyecto, se presentan las mejoras obtenidas en el cuadro de control que se tenía hasta la última revisión (se han anexado más indicadores se presentan los mismos del principio para poder realizar un comparativo tomando los datos del último año). Al igual que en la tabla anterior en la izquierda de la columna los datos reales obtenidos hasta la última muestra y en la derecha los objetivos planteados al inicio del proyecto. 


\section{CONCLUSIÓN}

La aplicación de los EAD en las organizaciones resultan una poderosa herramienta en la búsqueda de la tan ansiada productividad; sin embargo, para lograr su aplicación requiere de un programa bien establecido, un compromiso claro tanto de la alta dirección como de todos los involucrados y el contar con herramientas concretas que permitan su monitoreo e identificar áreas de oportunidad (instrumentos de medición, cursos, indicadores, mapeo de procesos). El aplicar los EAD requiere de tiempo y presupuesto por lo que no resultan una herramienta que se pueda aplicar en un tiempo breve (el tiempo dependerá de la madurez de cada organización y los pasos que se sigan para su aplicación), por lo que de realizar el proyecto en forma concreta y con un liderazgo definido en forma clara y abierta se pueden obtener grandes beneficios y coadyuvar a lograr no solo la productividad, sino además, a cimentar el futuro de la organización en general al comprender que las empresas que sobrevivirán en este nuevo entorno competitivo son aquellas que logren obtener conjuntar el capital intelectual y la pasión por el compromiso de todos sus integrantes en pro de los objetivos comunes de su organización.

\section{REFERENCIAS}

Bauer, J. (2002). A longitudinal evaluation of the impact of organizational structure on role ambiguity and work group performance. University of Sarasota.

Davis, K. y Newstrom, J. (2002). Comportamiento humano en el trabajo ( $11^{a}$ ed.). México: McGraw Hill.

Furnham, A. y Newstrom, J. (2002). Comportamiento humano en el trabajo ( $11^{a}$ ed.). México: McGraw Hill.

Martínez, J. (mayo-agosto, 2001). Símbolos de poder en las organizaciones y sus repercusiones. Hitos de ciencias económico administrativas, 7(18).

McShane, S. y Von, M. (2005). Organizational behavior: Emerging realities for the workplace revolution ( $3^{a}$ ed.). New York: McGraw-Hill.

Gordon, J. (1997). Comportamiento organizacional (5a ed.). México: Prentice Hall.

Robbins, S. (1998). La administración en el mundo de hoy (1 ${ }^{a}$ ed.). México: Pearson Educación.

TABLA II. CUADRO DE CONTROL-2

\begin{tabular}{|c|c|c|c|c|c|c|c|c|c|c|}
\hline \multirow{2}{*}{$\frac{\text { Tema }}{\text { Rotación del personal en \% }}$} & \multicolumn{2}{|c|}{$\mathrm{RH}$} & \multicolumn{2}{|c|}{$\mathrm{F}$} & \multicolumn{2}{|c|}{$P$} & \multicolumn{2}{|c|}{ L } & \multicolumn{2}{|c|}{$M$} \\
\hline & 0 & 2 & 0 & 1 & 5 & 20 & 3 & 20 & 4 & 10 \\
\hline $\begin{array}{l}\text { Mermas identificadas de sus } \\
\text { recursos asignados en \% }\end{array}$ & 3 & 2 & 1 & 1 & 6 & 5 & 4 & 5 & 6 & 5 \\
\hline Número de accidentes & 0 & 0 & 0 & 0 & 1 & 0 & 3 & 0 & 1 & 0 \\
\hline Reclamación de calidad & 0 & 0 & 0 & 0 & 6 & 0 & 4 & 0 & 8 & 0 \\
\hline AOL comunicación & A & A & A & A & M & A & A & A & A & $A$ \\
\hline $\begin{array}{l}\text { AOL capacidad para } \\
\text { relacionarse efectivamente }\end{array}$ & A & A & A & A & M & $A$ & A & A & A & $A$ \\
\hline AOL autocontrol & A & A & A & A & A & A & M & A & M & $A$ \\
\hline $\begin{array}{l}\text { AOL abierto a nuevas } \\
\text { experiencias }\end{array}$ & M & A & M & $A$ & M & $A$ & A & $A$ & A & $A$ \\
\hline AOC liderazgo & A & A & A & A & A & A & A & A & M & A \\
\hline AOC Comunicación & A & A & A & $A$ & M & $A$ & A & A & M & $A$ \\
\hline $\begin{array}{l}\text { AOC satisfacción hacia el } \\
\text { cambio }\end{array}$ & A & $A$ & A & $A$ & A & $A$ & A & $A$ & M & $A$ \\
\hline AOC trabajo en equipo & A & A & A & $A$ & A & $A$ & A & $A$ & A & $A$ \\
\hline
\end{tabular}

